\title{
The Elusive but Painful Subtalar Joint in Rheumatoid Arthritis
}
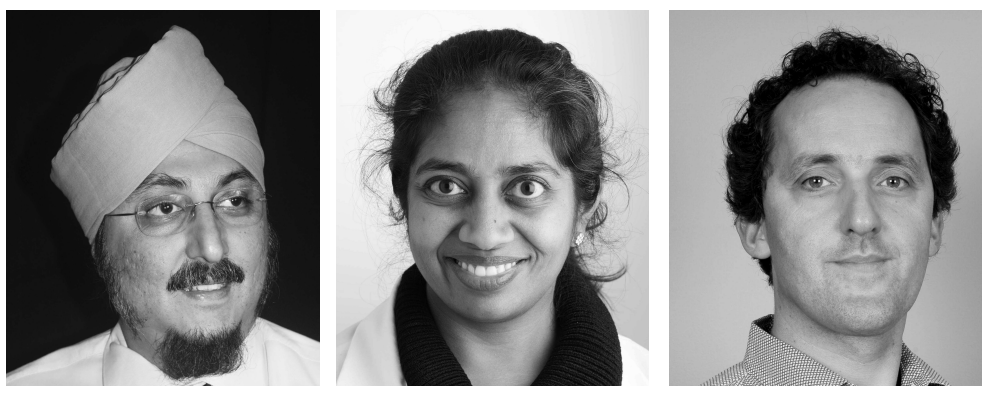

Foot pain is common in rheumatoid arthritis (RA), from forefoot involvement in early RA to later involvement of the hind foot. Foot and ankle involvement can lead to reduced walking distance and affect quality of life $^{1}$. The prevalence of hind foot pain was estimated at $17 \%$ in early RA and up to $52 \%$ in patients with established $\mathrm{RA}^{2}$. Left untreated, inflammation of the subtalar joint can lead to progressive eversion of the joint, posterior tibial tendon tendinopathy, and collapse of the medial $\mathrm{arch}^{3}$. Clinically, pain from the joint is often reported in the lateral hind foot.

Anatomically, the talus forms the mechanical gateway from the leg to the foot ${ }^{4}$. Its superior surface articulates with the fibula and tibia. A complex articulation is formed between the talus, calcaneus, and navicular bone inferiorly and anteriorly ${ }^{4,5}$. The subtalar joint is functionally a single joint that, anatomically, is divided into an anterior part, the talocalcaneonavicular joint or anterior subtalar joint (ASTJ) and a posterior part, the talocalcaneal joint or posterior subtalar joint (PSTJ $)^{4,5}$. The ASTJ is formed by the navicular bone and the head of the talus anteriorly and the anterior and middle facets of the talus and the calcaneus (sustentaculum tali) inferiorly (Figure 1a and 1b). The spring ligament supports the joint inferiorly. The talonavicular joint and anterior talocalcaneal joint therefore form a continuity and together will be referred to as the ASTJ ${ }^{4,5}$. The ASTJ is separated from the PSTJ by the sinus tarsi and tarsal canal with several strong ligaments within the sinus tarsi. The PSTJ consists of the posterior facets of the talus and the calcaneus ${ }^{4,5}$. It is difficult to assess clinically but the various components can well be assessed sonographically. Several approaches have been suggested. In a recent publication focused on the anatomic and sonographic description of the subtalar joint in adults, a set of 4 scans for the subtalar joint has been proposed ${ }^{5}$. The posterior subtalar joint has a separate joint capsule that usually does not communicate with the anterior articulation ${ }^{6}$. Both joints have a common dependent axis of motion akin to the hinges on a door ${ }^{4}$. The medial and lateral ankle tendon compartments are in close proximity to the subtalar joint. The synovial sheaths of the flexor hallucis tendon may communicate with the tibiotalar or subtalar joint. In patients with RA, the subtalar joint may also communicate with the tibiotalar joint ${ }^{6,7}$. It is noteworthy that symptoms may arise from these tendon structures as well as the tibiotalar and subtalar joint.

Physical examination of the subtalar joint is limited to eliciting range of eversion and inversion and seeing whether this is painful. Early progression of disease is difficult to ascertain with plain radiography and the opportunity to treat underlying disease and preventing future disability may be missed $^{3}$. Sectional imaging with magnetic resonance imaging can provide information about synovitis as well as bone and cartilage architecture, but is expensive. Musculoskeletal ultrasound (US) has been used in the past to evaluate for subtalar joint effusion, but cannot inform on the internal structure of the joint. Of note, it reveals only synovial fluid and synovial hypertrophy in the bony margins of the articulations as opposed to other joints, where available sonographic windows inform on wider aspects of the joint structure.

In this issue of The Journal, Bruyn, et al report on reliability of sonography of the subtalar joint using consensus-driven definitions ${ }^{8}$. This is an interesting extension of the above-mentioned publication on the normal US anatomy of the subtalar joint ${ }^{5}$. Consensus definitions were reached after a literature review and Delphi process. The manuscript reports on a reliability exercise in 10 patients with established RA and hind foot pain. Sonographers consisted of 10 rheumatologists and 2 podiatrists. The investigators are commended on their efforts to standardize subtalar joint evaluation with sonography, but the nomenclature used by Bruyn, et al is not entirely clear ${ }^{8}$. The authors state that the consensus was to exclude the talocalcaneonavicular joint, which is equivalent to the ASTJ, and assess the talocalcaneal joint only, which one has to assume then is referring to PSTJ. The PSTJ was assessed from a posterolateral scan, but then a medial scan was performed by positioning the probe on the sustentaculum tali and moving it anteriorly as well as posteriorly. The anteromedial scan as shown in Figure $2 b$ of Bruyn,

See US of subtalar synovitis, page 351

Personal non-commercial use only. The Journal of Rheumatology Copyright @ 2019 . All rights reserved. 

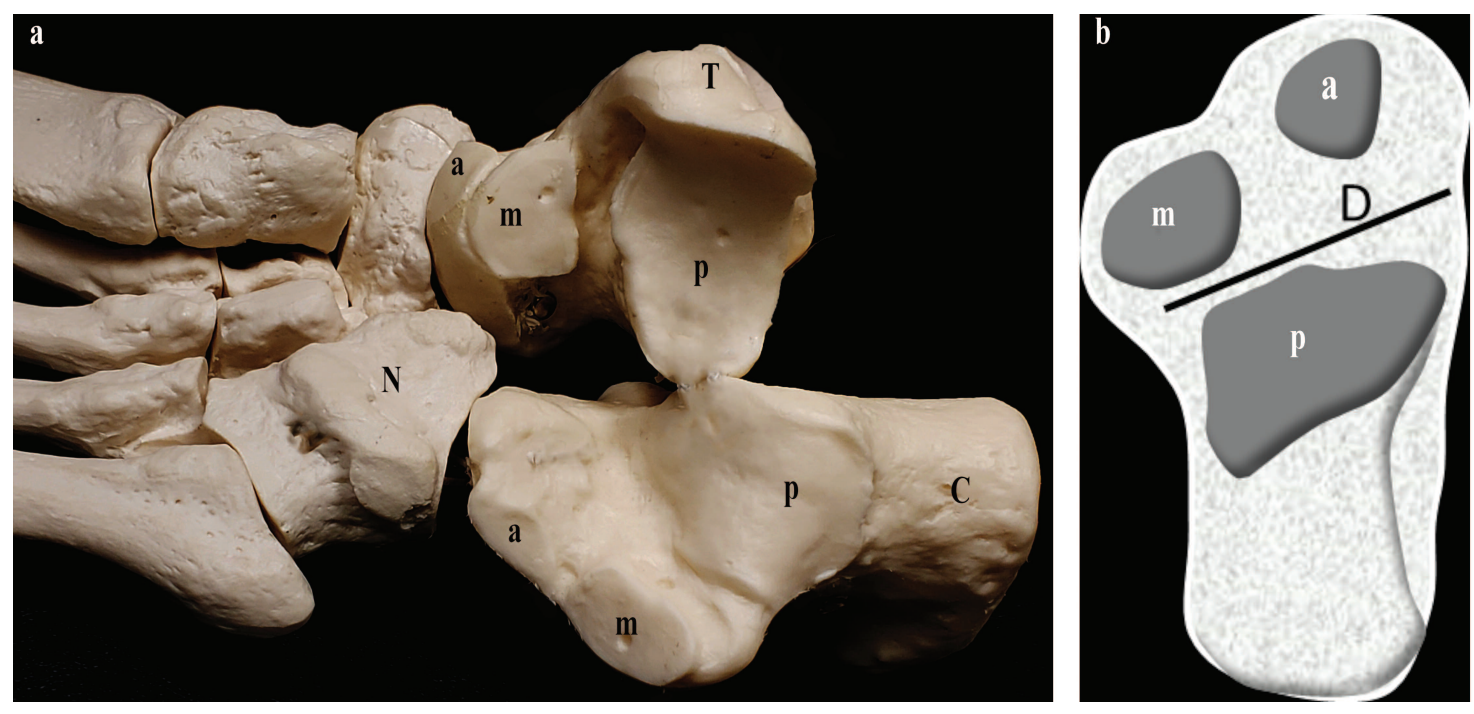

Figure 1. (a) Disarticulated talus (T) and calcaneus (C) demonstrating posterior facet articulations (p), middle facet articulations (m), anterior facet articulations (a). Navicular bone (N). (b) Schematic of superior calcaneal surface depicting separation of anterior and posterior subtalar joints by the tarsal canal and sinus tarsi (Line D). a: anterior facet; m: middle facet on top of sustentaculum tali; p: posterior facet.

et al will examine the talocalcaneonavicular joint or ASTJ ${ }^{8}$. The posteromedial scan in Figure $2 b$ in contrast will analyze the PSTJ from medial. As opposed to their statement, the authors were in fact examining the talonavicularcalcaneal joint or ASTJ as well.

The technique described in Mandl, et $a l^{5}$ to study the ASTJ from medial is slightly complicated, involving a rotation of the probe. The technique described by Bruyn, et $a l^{8}$ of positioning the probe on the talus and sustentaculum tali and then moving anteriorly might be easier and sufficient to examine the ASTJ medially. It is nevertheless important to clearly recognize that this maneuver will examine the ASTJ, and future studies will have to evaluate whether the assessment of the ASTJ through the talonavicular view may add additional information in RA.

On the lateral scan, sweeping the probe into the sinus tarsi was not done. Gadolinium enhancement of the sinus tarsi has been shown to correlate highly with subtalar joint compartment involvement ${ }^{9}$. When severe, synovitis can be seen in the sinus tarsi, which anatomically abuts the posterior facet (Figure 2). Further, when cadaveric posterior subtalar joints are injected through the sinus tarsi, posterior and lateral recess distension could be demonstrated by sonography ${ }^{10}$. Using computed tomography arthrography, and injection through the sinus tarsi approach, the same authors demonstrated a similar distribution of contrast material as with the sonographic approach and the contrast material did not communicate with the middle facet articulation. Thus, the technique proposed by Bruyn, et $a l^{8}$ may not comprehensively examine the posterior subtalar joint. Positioning the probe over the sinus tarsi and then sliding posteriorly may be easier technically because it can be easily identified and allow for a rapid and comprehensive assessment of the PSTJ from lateral, knowing that in healthy subjects the synovial recess should not extend into the sinus tarsi, but in patients it may well do so.

No concurrent MRI was performed. Three different machines from the same manufacturer were used, with standardized settings. Power Doppler and synovial hypertrophy were scored semiquantitatively, while joint effusion was scored dichotomously. There was fair to moderate interobserver agreement for synovial hypertrophy and Power Doppler and poor agreement for joint effusion. Although the separation of joint fluid from synovial hypertrophy was initially suggested to alleviate poor machine Doppler sensitivity, operationally it has not been reliable. The most reliable scans were the posterolateral scans for synovial hypertrophy and Power Doppler.

It may be instructive to compare the study by Lanni, et al in 50 patients with juvenile idiopathic arthritis, in which the subtalar joint was examined medially, laterally including sinus tarsi, and posteriorly ${ }^{11}$. Among the 2 investigators, the intraobserver and interobserver reliability were good to excellent. The lateral scans were felt to be the most prevalent for synovitis, but this may be, as also stated by Bruyn, et al, due to the more superficial location of the joint recess $^{8}$. In both studies the question arises of whether sensitivity for the detection of especially Doppler signals may be better by adjusting settings for each view and not using the same settings for all views. 


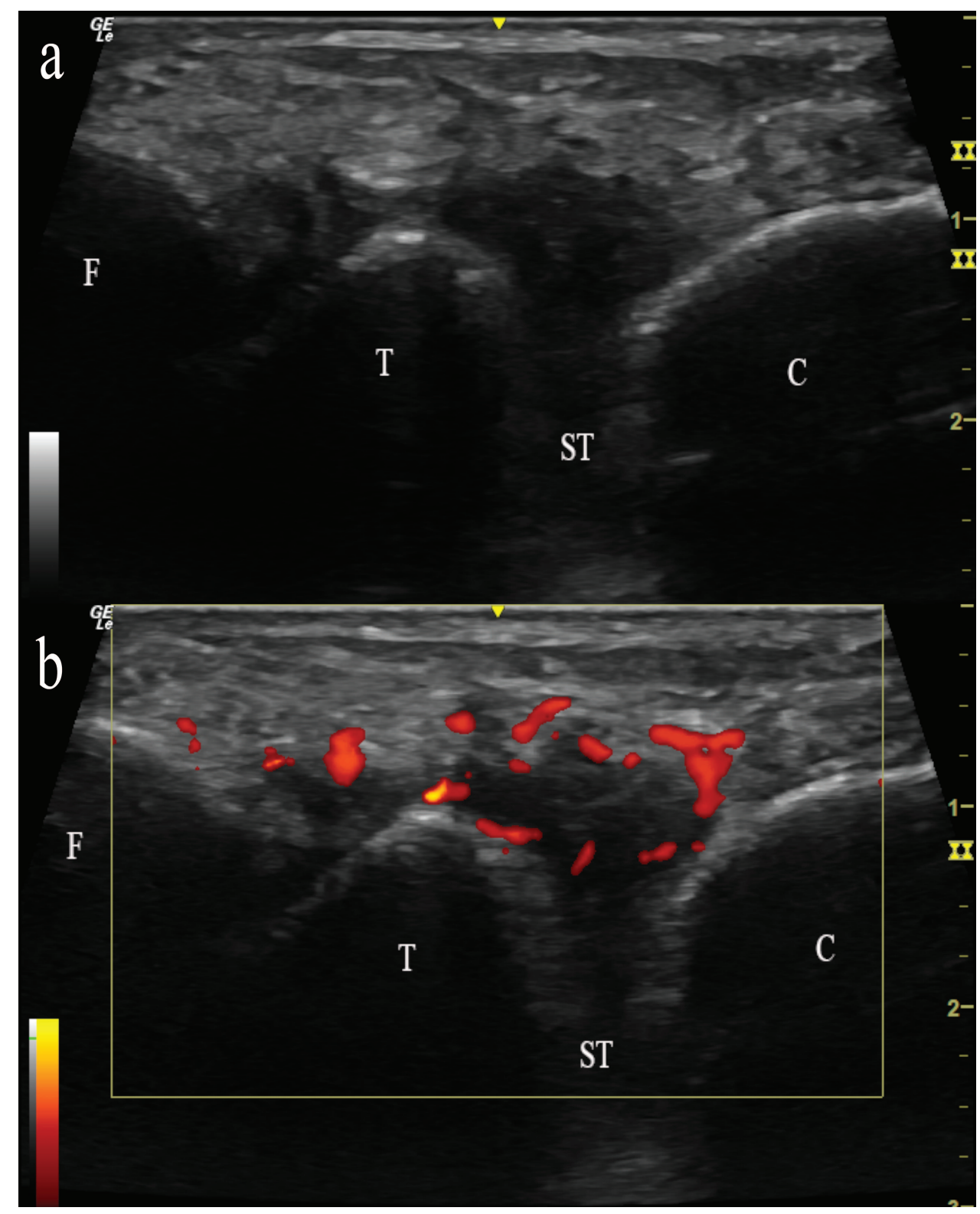

Figure 2. Synovitis demonstrated seeping into the sinus tarsi (ST) and showing continuity with synovitis in the tibiotalar joint. F: fibula; T: talus; C: calcaneus.

The article by Bruyn, et al represents an important step to address the subtalar joint, a forgotten yet functionally very relevant joint in $\mathrm{RA}^{8}$. Future studies will need to extend to a truly comprehensive assessment of this joint and should include the surrounding joints and tendons. From a diagnostic point of view, US can assist in not only pinpointing the source of pain but can also offer guidance for injections. As always, careful attention to anatomy is mandatory.

GURJIT S. KAELEY ${ }^{(D)}$, MRCP, RhMSUS, University of Florida College of Medicine, Jacksonville, Florida;
VEENA K. RANGANATH, MD, MS, RhMSUS, University of California at Los Angeles, Los Angeles, California, USA;

JOHANNES ROTH, MD, PhD, FRCPC, RhMSUS, University of Ottawa,

Ottawa, Ontario, Canada.

Address correspondence to Dr. G.S. Kaeley, 653-1 West Eighth St., LRC 2nd Floor L-14, Jacksonville, Florida 32209-6561, USA.

E-mail: drgurj@gmail.com

\section{REFERENCES}

1. Wilson O, Hewlett S, Woodburn J, Pollock J, Kirwan J. Prevalence, impact and care of foot problems in people with rheumatoid 
arthritis: results from a United Kingdom based cross-sectional survey. J Foot Ankle Res 2017;10:46.

2. Grondal L, Tengstrand B, Nordmark B, Wretenberg P, Stark A. The foot: still the most important reason for walking incapacity in rheumatoid arthritis: distribution of symptomatic joints in 1,000 RA patients. Acta Orthop 2008;79:257-61.

3. Chan PS, Kong KO. Natural history and imaging of subtalar and midfoot joint disease in rheumatoid arthritis. Int J Rheum Dis 2013;16:14-8.

4. Rockar PA Jr. The subtalar joint: anatomy and joint motion. J Orthop Sports Phys Ther 1995;21:361-72.

5. Mandl P, Bong D, Balint PV, Hammer HB, Miguel M, Naredo E, et al; Anatomy for the Image Study Group. Sonographic and anatomic description of the subtalar joint. Ultrasound Med Biol 2018; 44:119-23.

6. Beaudet F, Dixon AS. Posterior subtalar joint synoviography and corticosteroid injection in rheumatoid arthritis. Ann Rheum Dis 1981;40:132-5.
7. Hug G, Dixon AS. Ankle joint synoviography in rheumatoid arthritis. Ann Rheum Dis 1977;36:532-9.

8. Bruyn GA, Siddle HJ, Hanova P, Costantino F, Iagnocco A, Delle Sedie A, et al. Ultrasound of subtalar joint synovitis in patients with rheumatoid arthritis: results of an OMERACT reliability exercise using consensual definitions. J Rheumatol 2019;46:351-9.

9. Bouysset M, Tebib J, Tavernier T, Noel E, Nemoz C, Bonnin M, et al. Posterior tibial tendon and subtalar joint complex in rheumatoid arthritis: magnetic resonance imaging study. J Rheumatol 2003;30:1951-4

10. Smith J, Maida E, Murthy NS, Kissin EY, Jacobson JA. Sonographically guided posterior subtalar joint injections via the sinus tarsi approach. J Ultrasound Med 2015;34:83-93.

11. Lanni S, Bovis F, Ravelli A, Viola S, Magnaguagno F, Pistorio A, et al. Delineating the application of ultrasound in detecting synovial abnormalities of the subtalar joint in juvenile idiopathic arthritis. Arthritis Care Res 2016;68:1346-53.

J Rheumatol 2019;46:333-6; doi:10.3899/jrheum.181156 